\title{
Vagus nerve stimulation in pregnant rats and effects on inflammatory markers in the brainstem of neonates
}

\author{
Allison Judkins ${ }^{1}$, Rhaya L Johnson ${ }^{2}$, Samuel T Murray ${ }^{2}$, Steven M Yellon ${ }^{2}$ and Christopher G. Wilson ${ }^{2}$
}

BACKGROUND: Vagus nerve stimulation (VNS) is an Food and Drug Administration-approved method delivering electrical impulses for treatment of depression and epilepsy in adults. The vagus nerve innervates the majority of visceral organs and cervix, but potential impacts of VNS on the progress of pregnancy and the fetus are not well studied.

METHODS: We tested the hypothesis that VNS in pregnant dams does not induce inflammatory changes in the cardiorespiratory control regions of the pups' brainstem, potentially impacting the morbidity and mortality of offspring. Pregnant dams were implanted with stimulators providing intermittent low or high frequency electrical stimulation of the subdiaphragmatic esophageal segment of the vagus nerve for 6 7 days until delivery. After birth, we collected pup brainstems that included cardio-respiratory control regions and counted the cells labeled for pro-inflammatory cytokines (interleukin (IL)-1 $\beta, \quad I L-6$, tumor necrosis factor-a) and high mobility group box 1 .

RESULTS: Neither pup viability nor number of cells labeled for pro-inflammatory cytokines in nucleus tractus solitarii or hypoglossal motor nucleus was impaired by VNS. We provide evidence suggesting that chronic VNS of pregnant mothers does not impede the progress or outcome of pregnancy.

CONCLUSION: VNS does not cause preterm birth, affect wellbeing of progeny, or impact central inflammatory processes that are critical for normal cardiovascular and respiratory function in newborns.

V agus nerve stimulation (VNS) has wide-ranging clinical applications, including Food and Drug Administrationapproved treatments for epilepsy and depression in adults. Over 75000 patients have been implanted with VNS devices (1). VNS is currently being investigated as a clinical treatment for sepsis and rheumatoid arthritis, because it suppresses peripheral inflammation and may be important in modulating neuroinflammation $(2,3)$. The effects of VNS have been correlated with serum tumor necrosis factor (TNF) concentrations as a way to assess the efficacy of VNS to treat endotoxemia (4). The anti-inflammatory effect also seems to depend upon reduction of inflammation via descending cholinergic efferent output $(4,5)$. Although VNS has therapeutic value in adult patients, its impact on pregnancy, birth, and on fetal development and well-being remain largely unknown. Owing to the inevitability of pregnancy in women undergoing VNS treatment, the impact of this therapy on pregnancy and fetal well-being is an important consideration - particularly with the increasing number of vagus nerve stimulators implanted over the past decade with expanding Food and Drug Administration approval (1). Case reports of VNS therapy in pregnant women found no adverse effects on pregnancy or the postpartum neonate $(6,7)$, though these studies did not include physiological end points or extend to the use of animal models.

The vagus nerve interconnects the medulla, the heart, lungs, stomach, and other viscera, including the colon and female reproductive tract (8), thus any change in vagal activity would be expected to have an impact on these systems. Approximately $80 \%$ of vagus fibers carry afferent information to the central nervous system while $20 \%$ of the vagus is efferent information to the periphery (9). Though the uterus is virtually denervated during pregnancy, cervix remodeling involves parasympathetic innervation from the vagus and pelvic nerves to the cervix (10), in part via brain nitric oxide synthase and calcitonin gene-related peptide fibers (11). These sensory neuropeptides mediate inflammatory responses in other tissues (11). We have previously shown that vagus nerve transection reduces the presence of macrophages in the cervix but also resulted in distension of the bladder and stomach in our previous work-suggesting that descending vagal input is key to normal cervical function (12) as well as autonomic tone to the viscera. As vagus nerve transection in pregnant rats has such a significant impact, investigating the effect of VNS on the progression of pregnancy and inflammatory processes in the fetus is warranted and understanding the role of VNS on pregnancy and pup outcome are the major motivators for this work.

VNS may be an effective treatment during pregnancy for infections that result from a compromised cervix immune barrier, as may occur with chorioamnionitis or premature rupture of fetal membranes (13-16), which exposes the developing fetus to pro-inflammatory cytokines and is

\footnotetext{
${ }^{1}$ Departments of Pediatrics and Basic Sciences, Division of Physiology, Loma Linda University School of Medicine, Loma Linda, CA; ${ }^{2}$ Lawrence D. Longo Center for Perinatal Biology, Loma Linda University School of Medicine, Loma Linda, CA. Correspondence: Christopher G. Wilson (cgwilson@llu.edu)

Received 19 May 2017; accepted 25 September 2017; advance online publication 22 November 2017. doi:10.1038/pr.2017.265
} 
associated with neonatal morbidity and brain injury. These cytokines are part of the developmental signals that regulate prenatal neural connectivity (17) in specific brain regions, and there is limited evidence to indicate a reduced size of brainstem nuclei following systemic treatment with endotoxin, a prenatal inflammatory agent (18). Inflammation in these regions causes impaired breathing responses to hypoxia in neonatal rat pups $(19,20)$. Thus in this study, we tested the hypothesis that maternal VNS would interfere with parturition and alter the level of pro-inflammatory cytokines in cardio-respiratory regions of the brainstem. We tested this hypothesis by using immunohistochemistry to label for proinflammatory cytokines and the transcription factor high mobility group box 1 (HMGB1). Our objective was to determine whether chronic VNS impacts morbidity and mortality in infants born to mothers with implanted vagal stimulators.

\section{METHODS}

Adult Long Evans rats (Harlan, Indianapolis, IN) were individually housed in a temperature- and humidity-controlled vivarium with a 12-h light/dark cycle with food and water ad libitum. Animal care and usage followed the National Institutes of Health guidelines and all experimental procedures were approved by the LLU Institutional Animal Care and Use Committee (IACUC).

\section{Dam Surgery and VNS}

Two days after acclimatization to the vivarium, we performed surgery on pregnant dams at day 16 postbreeding, under $2-4 \%$ isoflurane-oxygen gas anesthesia (Figure 1, overview of study design and timeline). A midline incision was made below the rib cage to expose the subdiaphragmatic vagus nerve, which was then separated from the connective sheath and vasculature to obtain an accessible length of approximately $1 \mathrm{~cm}$. The vagus nerve was isolated at the subdiaphgramatic portion of the esophagus following previously described procedures (12). After gentle, blunt dissection, a glass hook and fine forceps were used to separate the posterior vagal trunk and isolate a length of nerve sufficient to allow electrodes to encircle the nerve. VNS stimulators were fabricated by Harald Stauss Scientific and activated via a Hall Effect switch. We used the RNS model stimulator (http://haraldstauss.com/HaraldStaussScientific/default. html) to maximize current density over the course of the stimulation period. The electrode-wrapped vagus nerve was covered with a thin film of a silicone-based sealant (Kwik-sil, WPI, Sarasota, FL) to isolate and insulate the stimulation area. The electrode wires were connected to a battery-powered, implantable stimulator unit that was inserted into the abdominal cavity. Following surgery, the abdomen wall was sutured and the skin secured with clips. Pregnant dams were randomly assigned to groups in which the implanted VNS device was set to deliver 5 or $1,000 \mathrm{~Hz}$ at $1 \mathrm{~mA}$ amplitude with $500 \mu$ s pulse width or no current (Sham). Our stimulation parameters were based on parameters commonly used in clinical practice and preclinical studies assessing the effect of vagal nerve stimulation on selective fiber blockade $(9,21)$.

Stimulation duration was $30 \mathrm{~min}$ (on-time) at the given frequency for that animal and was followed by an off-time of $5.5 \mathrm{~h}$ repeated $4 \times$ per day. The programmed frequencies were confirmed each day using a digital AM/FM radio (Sony Corp of America, New York, NY, USA). Weight and food consumption were monitored daily to assess well-being of the dams and verify the stimulation regimen. Thus the pups assessed for this project were exposed to either 5 or $1,000 \mathrm{~Hz}$ over 5-6 days post-VNS stimulator implantation as dams were allowed to continue in the study until parturition (typically gestational day 21-22 in rats).

Processing of Brainstems, Immunohistochemistry, and Analyses Within 12 hours of delivery, dams were asphyxiated with $\mathrm{CO}_{2}$. Pups were deeply anesthetized with isofluorane and their brainstems were removed, rinsed with chilled saline, and stored in $4 \%$ paraformaldehyde for $24 \mathrm{~h}$ before transfer to $30 \%$ sucrose for $48 \mathrm{~h}$ to provide cryoprotection. Brainstems were frozen in Tissue-Tek OCT and sectioned at $20 \mu \mathrm{m}$ on a Leica CM 3050 S (Buffalo Grove, IL, USA) cryostat. Sections of brainstem were stained using immunohistochemistry with selective antibodies for interleukin (IL)-6 (1:100 sc1265-r, Santa Cruz, Dallas, TX, USA), TNF $\alpha$ (1:100 ab6671, Abcam, Cambridge, MA, USA), IL-1 $\beta$ (1:100 ab9722, Abcam), and HMGB-1 (1:1,000 ab18256, Abcam) as previously described (22). The number of cells expressing IL-6, TNF $\alpha$, IL-1 $\beta$, and HMGB1 protein were counted using a Zeiss Axio Imager A1 (Buffalo Grove, NY, USA) and unbiased stereology (Stereologer2000, Stereology Resource Center). All cell counts were normalized to volume assessed to account for variability in tissue morphology across sections. Photographs were acquired within Stereologer and postprocessing was carried out using GIMP (http://gimp.org) and Adobe Illustrator CS6 (San Jose, CA, USA). We analyzed all data using one-way analysis of variance with the Duncan post hoc test (SPSS, v22, IBM, Armonk, NY, USA) with a $P<0.05$ considered significant.

\section{RESULTS}

\section{VNS Effects on Pregnancy, Parturition, and Pup Viability}

Neither surgery nor VNS interfered with either the progression of pregnancy or the parturition process. Pregnancy proceeded to term and litters had delivered in Sham $(n=4)$ and VNS $(5 \mathrm{~Hz} n=10,1,000 \mathrm{~Hz} n=11)$ groups by day $21-22$ postbreeding (see Figure 1 for timing of VNS surgery and

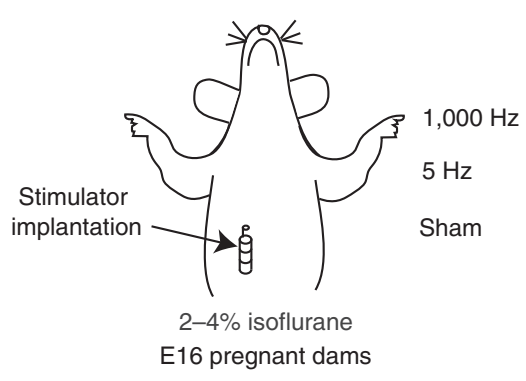

Killing

and neonate brain tissue collected

Figure 1. Vagus nerve stimulation protocol. Vagus nerve stimulation protocol and timeline for experiments. In each treatment group: $1,000 \mathrm{~Hz}, 5 \mathrm{~Hz}$ and Sham, we implanted stimulators with helical electrode contacts encircling the subdiaphragmatic, esophageal branch of the vagus nerve at embryonic day 16 (E16) in pregnant dams. Pregnancies proceeded to birth and the pups were killed and the brainstems removed within $12 \mathrm{~h}$ after birth. 


\section{Articles | Judkins et al.}

exposure). Pups were viable-breathing, with vigorous movement and vocalization when held. The pups were pink and had milk present in their stomachs when harvest of tissue began, consistent with the metrics used to assess neonatal well-being using an Apgar scale (23). This is consistent with data seen from vagus nerve transection pups from dams with transected vagus nerve pups from previous work (12). Table 1 summarizes the gestational age at delivery, number of live pups per litter, number of pups resorbed in utero, and postpartum pup mortality for each treatment group $(5 \mathrm{~Hz}$, $1,000 \mathrm{~Hz}$, and Sham).

\section{Brainstem Cell Counts}

We used unbiased stereology to count the number of specific stained cells in pups from each group in the nucleus tractus solitarii from -12.0 to $-14.7 \mathrm{~mm}$ (relative to Bregma), which encompasses the critical cardio-respiratory control regions in the brainstem. In all treatment groups, we counted $>500$ cells across the nucleus tractus solitarii. Compared with Sham controls, the number of IL-1 $\beta$ (Figure 2, panels a1-c1), IL-6 (Figure 2, panels a2-c2), TNF $\alpha$ (Figure 2, panels a3-c3), and HMGB1 (Figure 2, panels a4-c4) labeled were not significantly different in pups from dams that received the 5 or $1,000 \mathrm{~Hz}$ VNS. Table 2 shows the summary counts (mean $\pm \mathrm{SD}$ ) and $P$-value for each marker. Figure 3 shows summary histograms with mean cell counts for Il-1 $\beta$ (panel a), IL-6 (panel b), TNF $\alpha$ (panel c), and HMGB1 (panel d) $( \pm \mathrm{SD})$ for all treatments and stains.

In addition to the canonical, early-onset pro-inflammatory cytokines, we stained for HMGB1, due to its putative antiinflammatory role. We saw a non-significant increase in HMGB1 staining in the nucleus tractus solitarii in the $1,000 \mathrm{~Hz}$ stimulation group when compared with $5 \mathrm{~Hz}$ and Sham treatments, which is opposite of the trend seen with IL-6 and TNF $\alpha$.

\section{DISCUSSION}

In this study, we found that maternal VNS does not significantly increase the expression of pro-inflammatory cytokines in the cardio-respiratory control regions of the medulla, does not interfere with the parturition process, does not cause preterm birth, nor negatively impacts viability of the pups born to VNS-treated dams. Because we saw no ill-effects of maternal VNS, our data suggest that there is no impairment of the physiological processes essential for normal birth and viability of neonates. These findings support and extend observations in case reports describing the effectiveness of VNS therapy during pregnancies in which VNS has been used to treat depression and refractory epilepsy. The authors' of these reports found no impact on the timing of delivery of the mothers' infants $(6,24)$. An additional study of eight pregnancies in which the mothers received VNS for refractory epilepsy showed no adverse effects on either pregnancy or neonatal viability (7). Collectively, these findings support the suggestion that VNS
Table 1. VNS effects on pregnancy outcome and demographic features

\begin{tabular}{lccc}
\hline & $5 \mathrm{~Hz}$ & $1,000 \mathrm{~Hz}$ & Sham \\
\hline Gestational age at delivery & E22 & E21 & E22 \\
Liveborn pups & 10 & 11 & 4 \\
Number of pups resorbed in utero & 0 & 1 & 0 \\
Postpartum pup mortality & 0 & 1 & 0 \\
\hline
\end{tabular}

Gestational age at delivery, number of live pups, number of pups resorbed, and postpartum pup mortality are reported here.

may be included in therapeutic approaches to diseases during pregnancy.

The importance of these findings is underscored by the choice of physiologically relevant VNS characteristics. We used low frequency and current stimulation parameters that mimicked those seen in clinical use (25). Additionally, we used a higher stimulus frequency $(1,000 \mathrm{~Hz})$ that blocks a significant proportion of the afferent traffic carried via the vagus $(21,26)$. Neither VNS stimulus regimes resulted in significant changes in IL-1 $\beta$, IL- $6, \mathrm{TNF} \alpha$, or HMGB1 across our treatment groups. We focused on the early-onset cytokines and HMGB1 as they represent the "first responders" of inflammatory regulation. HMGB1 is a chromatinassociated protein that has a key role in transcription and regulation of gene expression. It has also been implicated in the anti-inflammatory response observed in other studies of VNS $(27,28)$. We also constrained our focus to the brainstem regions critical for cardio-respiratory control and implicated in neonatal morbidity and mortality $(29,30)$. In the brain, IL-6 is typically produced in response to the early upregulation of proinflammatory cytokines such as IL-1 $\beta$ via the nuclear factor- $\kappa \mathrm{B}$ pathway $(31,32)$. In addition to IL-6, TNF $\alpha$, and IL- $1 \beta$ are produced and released as part of the early inflammatory response (33). IL-1 $\beta$ is a key proinflammatory cytokine that can positively feedback to the nuclear factor- $\mathrm{\kappa B}$ (31) signaling cascade and exacerbate the production of pro-inflammatory cytokines to amplify the inflammatory response (34). TNF $\alpha$ triggers local inflammatory responses and serves as a co-factor for modulation of presynaptic release in the central nervous system (35) and the succeeding cascade of both pro- and anti-inflammatory cytokines and chemokines. The trophic role of $\mathrm{TNF} \alpha$ in modulation of brainstem autonomic circuits is not yet known but it has been shown to be necessary for appropriate synaptic formation (35). Work has previously been carried out in murine models looking at obesity-induced inflammatory changes in the expression of IL-6 in the hypothalamus (17), and this is an area of research that warrants further research.

VNS has been implicated in the modulation of inflammatory tone and been suggested as a therapy to alleviate the impact of chronic inflammation. VNS upregulates the expression of TNF $\alpha$ (5) systemically in non-pregnant adult murine models, and this has been shown to activate other cytokines, such as IL-1 $\beta$, and HMGB1 (4) which is implicated in both pro- and anti-inflammatory responses to VNS as part 

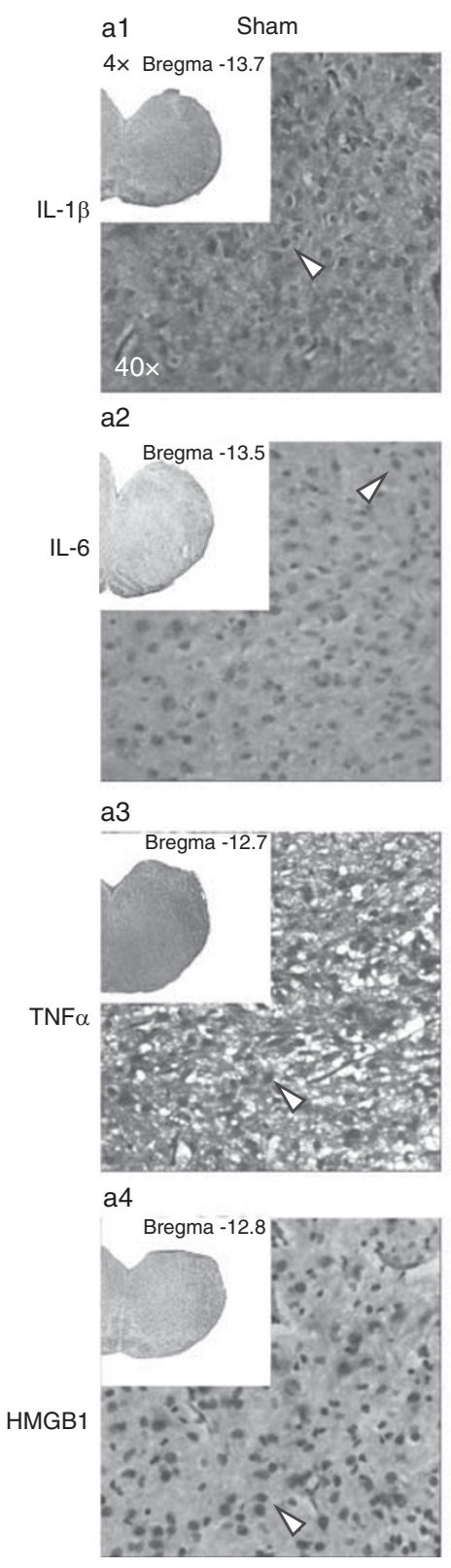

b1 $5 \mathrm{~Hz}$

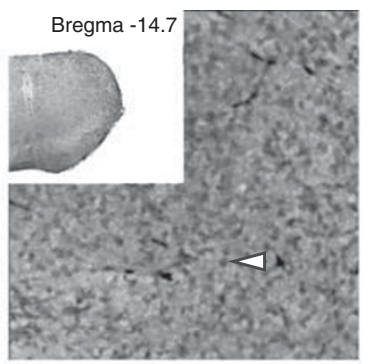

b2

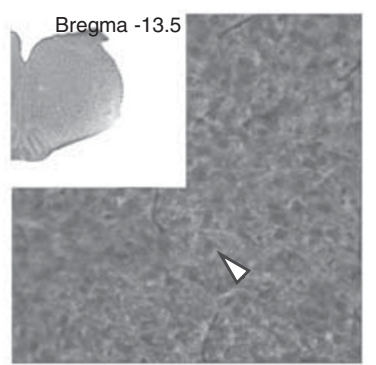

b3

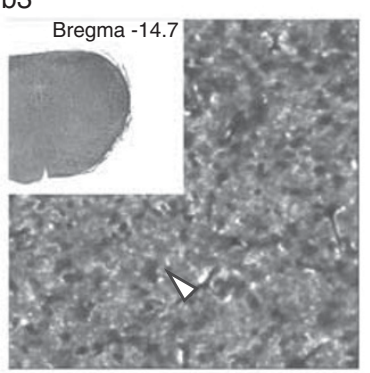

b4

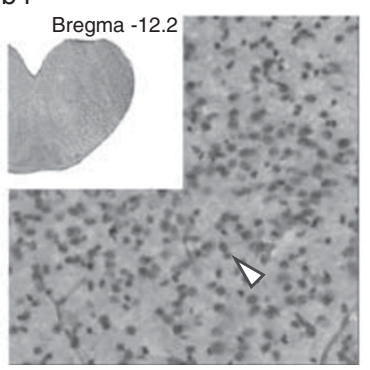

c1 $\quad 1,000 \mathrm{~Hz}$

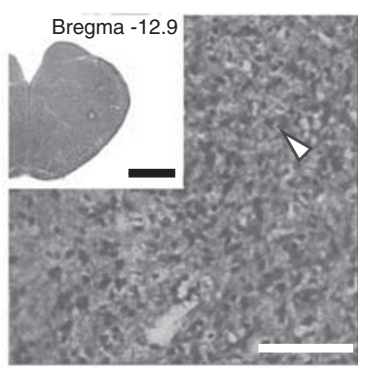

C2

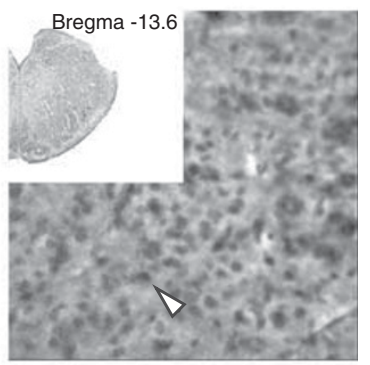

c3

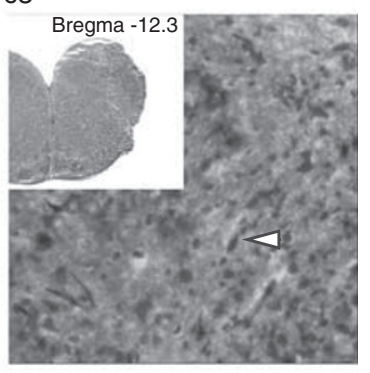

c4

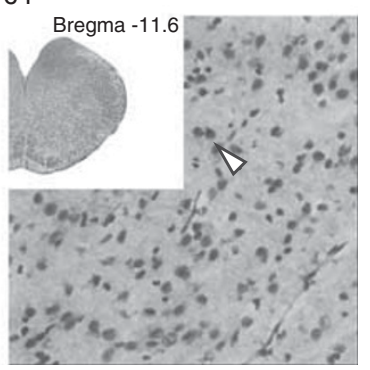

Figure 2. Immunohistochemistry staining for cytokines and high mobility group box 1 (HMGB1). From top to bottom, we show interleukin (IL)-1 $\beta$ (a1-c1), IL-6 (a2-c2), tumor necrosis factor-a (TNFa) (a3-c3), and HMGB1 (a4-c4) immuno-staining for each treatment (Sham, 5 Hz, 1,000 Hz). Each panel shows an inset photomicrograph $(4 \times)$ with the distance from Bregma and higher resolution photomicrograph at $40 \times$. Arrows indicate representative stained cells. Scale bar $=1 \mathrm{~mm}(4 \times)$ and $100 \mu \mathrm{m}(40 \times)$.

Table 2. Cell counts per volume across stimulation groups

\begin{tabular}{|c|c|c|c|c|}
\hline & $5 \mathrm{~Hz}$ & $1,000 \mathrm{~Hz}$ & Sham & $P$-value \\
\hline IL-1 $\beta$ & $5.19 \times 10^{-5} \pm 1.69 \times 10^{-5}$ & $3.91 \times 10^{-5} \pm 7.18 \times 10^{-6}$ & $4.34 \times 10^{-5} \pm 1.78 \times 10^{-5}$ & 0.154 \\
\hline IL-6 & $3.89 \times 10^{-5} \pm 1.06 \times 10^{-5}$ & $3.66 \times 10^{-5} \pm 1.05 \times 10^{-5}$ & $3.59 \times 10^{-5} \pm 1.47 \times 10^{-5}$ & 0.684 \\
\hline TNFa & $6.12 \times 10^{-5} \pm 2.97 \times 10^{-5}$ & $5.01 \times 10^{-5} \pm 6.97 \times 10^{-6}$ & $4.80 \times 10^{-5} \pm 1.32 \times 10^{-5}$ & 0.277 \\
\hline HMGB1 & $7.91 \times 10^{-5} \pm 1.25 \times 10^{-5}$ & $9.92 \times 10^{-5} \pm 1.28 \times 10^{-5}$ & $8.94 \times 10^{-5} \pm 2.73 \times 10^{-5}$ & 0.099 \\
\hline
\end{tabular}

HMGB1, high mobility group box 1; IL, interleukin; TNFa, tumor necrosis factor-a.

Each inflammatory marker and the mean number of cells expressing that marker are reported here \pm SD. 


\section{Articles | Judkins et al.}
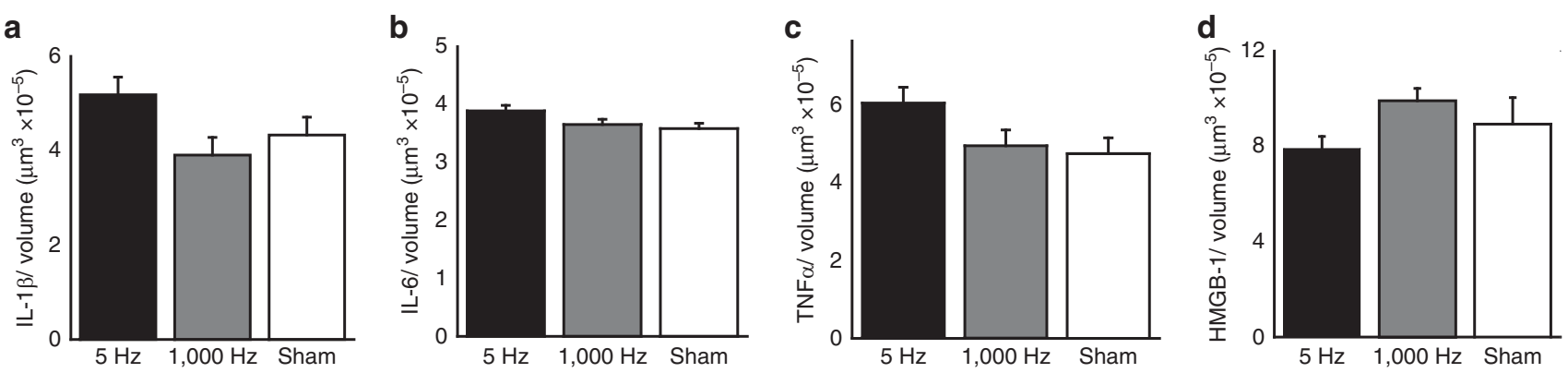

Figure 3. Summary histograms of unbiased stereology results for immunolabeling. All results are reported as the number of stained cells vs. stimulation parameter. Markers shown are interleukin (IL)-1 $\beta$ (a), IL-6 (b), tumor necrosis factor-a (TNFa) (c), and high mobility group box 1 (HMGB1) (d). Cell count numbers were divided by volume of area of interest to normalize for tissue size and then averaged (reported as average \pm SD). $N=3$ for all groups.

of hypothalamic-pituitary-adrenal axis activation $(36,37)$. IL-1 $\beta$ is the proto-typical early-onset pro-inflammatory cytokine and lipopolysccharide injection induces local expression of IL-1 $\beta$ in respiratory control centers of the brainstem $(19,20)$. Because these cytokines are upregulated very quickly and early in the inflammatory response, their expression in response to inflammation may provide an early indication of the efficacy of VNS in either promoting or blocking their expression and subsequent changes in inflammatory tone.

The work we report here suggests that VNS lacks significant negative impact on the production and release of cytokines and does not impair the timing of parturition or the viability of the newborn. An obvious limitation of our study is that we constrained our stimulus parameters to two sets (relatively low and high) and we did not employ animal models of inflammation or seizure to determine the efficacy of VNS as an intervention. Further studies will need to be performed to determine whether there are stimulus parameters for VNS that are inherently pro- and anti-inflammatory.

\section{Limitations of the Study}

Our work is innovative in assessing the role of VNS in a pregnant rat model but there are inherent limitations to our study. Because we used implantable stimulators with limited battery, we were limited in the current density available to block using kilohertz frequency alternating current. Other investigators have used anesthetized animal models to perform kilohertz frequency stimulation and show complete neural block of peripheral nerves at higher frequencies than we used in our experiments $(21,26,38-40)$. We used a maximum of $1,000 \mathrm{~Hz}$ for our stimuli after consulting with the vendor of our implantable stimulators to assure continuous stimulation over the entire experimental time period. Additionally, we did not assess anti-inflammatory markers; however, HMGB1 is implicated in both anti- and pro-inflammatory roles $(37,41)$. Future work will incorporate a more extensive panel of pro- and anti-inflammatory markers and a wider range of stimulus parameters to assess the impact that VNS has on immune state in both pregnant mothers and their offspring.
Further neurodevelopmental evaluation of neonates exposed to vagal nerve stimulation would provide a broader understanding of longer-term consequences of maternal VNS therapy. Assessing systemic C-reactive protein and procalcitonin as well as pro- and anti-inflammatory cytokines in babies born to these mothers would provide further information about the immune response evoked by VNS. Ultimately, the goal is to understand the effects of VNS on neuroinflammatory processes that contribute to the physiology of pregnancy, parturition, and fetal neurodevelopment. It may be possible to tune VNS to arrest or prophylactically prevent chorioamnionitis or other sources of inflammation in the mother and improve maternal and fetal outcome.

\section{STATEMENT OF FINANCIAL SUPPORT}

This work was supported in part by NIH HD954931. Imaging was performed in the Advanced Imaging and Microscopy Core with support from NSF Grant MRI-DBI 0923559 and the Loma Linda University School of Medicine Pediatrics Research Fund.

Disclosure: The authors declare no conflict of interest.

\section{REFERENCES}

1. Chakravarthy K, Chaudhry H, Williams K, Christo PJ. Review of the uses of vagal nerve stimulation in chronic pain management. Curr Pain Headache Rep 2015;19:54.

2. Meneses G, Bautista M, Florentino A, et al. Electric stimulation of the vagus nerve reduced mouse neuroinflammation induced by lipopolysaccharide. J Inflamm Lond Engl 2016;13:33.

3. Mihaylova S, Killian A, Mayer K, Pullamsetti SS, Schermuly R, Rosengarten B. Effects of anti-inflammatory vagus nerve stimulation on the cerebral microcirculation in endotoxinemic rats. J Neuroinflammation 2012;9:183.

4. Tracey KJ.. The inflammatory reflex. Nature 2002;420:853-9.

5. Borovikova LV, Ivanova S, Zhang $\mathrm{M}$, et al. Vagus nerve stimulation attenuates the systemic inflammatory response to endotoxin. Nature 2000;405:458-62.

6. Husain MM, Stegman D, Trevino K. Pregnancy and delivery while receiving vagus nerve stimulation for the treatment of major depression: a case report. Ann Gen Psychiatry 2005;4:16.

7. Ben-Menachem E. Vagus nerve stimulation for treatment of seizures? Arch Neurol 1998;55:231-2.

8. Collins JJ, Lin CE, Berthoud HR, Papka RE. Vagal afferents from the uterus and cervix provide direct connections to the brainstem. Cell Tissue Res 1999;295:43-54. 
9. Groves DA, Brown VJ. Vagal nerve stimulation: a review of its applications and potential mechanisms that mediate its clinical effects. Neurosci Biobehav Rev 2005;29:493-500.

10. Yellon SM. Contributions to the dynamics of cervix remodeling prior to term and preterm birth. Biol Reprod 2017;96:13-23.

11. Kirby LS, Kirby MA, Warren JW, Tran LT, Yellon SM. Increased innervation and ripening of the prepartum murine cervix. J Soc Gynecol Investig 2005;12:578-85.

12. Clyde LA, Lechuga TJ, Ebner CA, Burns AE, Kirby MA, Yellon SM. Transection of the pelvic or vagus nerve forestalls ripening of the cervix and delays birth in rats. Biol Reprod 2011;84:587-94.

13. Kallapur SG, Nitsos I, Moss TJM, et al. IL-1 mediates pulmonary and systemic inflammatory responses to chorioamnionitis induced by lipopolysaccharide. Am J Respir Crit Care Med 2009;179:955-61.

14. Chau V, McFadden DE, Poskitt KJ, Miller SP. Chorioamnionitis in the pathogenesis of brain injury in preterm infants. Clin Perinatol 2014;41: 83-103.

15. Perlman JM.. White matter injury in the preterm infant: an important determination of abnormal neurodevelopment outcome. Early Hum Dev 1998;53:99-120.

16. Burd I, Balakrishnan B, Kannan S. Models of fetal brain injury, intrauterine inflammation, and preterm birth. Am J Reprod Immunol 2012;67:287-94.

17. Sanders TR, Kim DW, Glendining KA, Jasoni CL. Maternal obesity and IL-6 lead to aberrant developmental gene expression and deregulated neurite growth in the fetal arcuate nucleus. Endocrinology 2014;155:2566-77.

18. Duncan JR, Cock ML, Scheerlinck J-PY, et al. White matter injury after repeated endotoxin exposure in the preterm ovine fetus. Pediatr Res 2002;52:941-9.

19. Balan KV, Kc P, Mayer CA, Wilson CG, Belkadi A, Martin RJ. Intrapulmonary lipopolysaccharide exposure upregulates cytokine expression in the neonatal brainstem. Acta Paediatr 2012;101:466-71.

20. Jafri A, Belkadi A, Zaidi SIA, Getsy P, Wilson CG, Martin RJ. Lung inflammation induces IL-1 $\beta$ expression in hypoglossal neurons in rat brainstem. Respir Physiol Neurobiol 2013;188:21-8.

21. Patel YA, Butera RJ. Differential fiber-specific block of nerve conduction in mammalian peripheral nerves using kilohertz electrical stimulation. J Neurophysiol 2015;113:3923-9.

22. Johnson RL, Murray ST, Camacho DK, Wilson CG. Vagal nerve stimulation attenuates IL- 6 and $\mathrm{TNF} \alpha$ expression in respiratory regions of the developing rat brainstem. Respir Physiol Neurobiol 2016;229:1-4

23. Antonelli MC, ed. Perinatal Programming of Neurodevelopment, 1st edn, 2015 edition. Springer Springer-Verlag, New York, NY, 2016.

24. Houser MV, Hennessy MD, Howard BC.. Vagal nerve stimulator use during pregnancy for treatment of refractory seizure disorder. Obstet Gynecol 2010;115:417-9.
25. Fang D, Shi S-Q, Shi L, et al. Direct electrical stimulation softens the cervix in pregnant and nonpregnant rats. Am J Obstet Gynecol 2015;212: 786.e1-9.

26. Patel YA, Saxena T, Bellamkonda RV, Butera RJ. Kilohertz frequency nerve block enhances anti-inflammatory effects of vagus nerve stimulation. Sci Rep 2017;7:39810.

27. Levy G, Fishman JE, Xu D, et al. Vagal nerve stimulation modulates gut injury and lung permeability in trauma-hemorrhagic shock. J Trauma Acute Care Surg 2012;73:338-42 discussion 342.

28. Bajbouj M, Merkl A, Schlaepfer TE, et al. Two-year outcome of vagus nerve stimulation in treatment-resistant depression. J Clin Psychopharmacol 2010;30:273-81.

29. Martin RJ, Wilson CG.. What to do about apnea of prematurity? J Appl Physiol 2009;107:1015-6.

30. Moorman JR, Lake DE, Ivanov PC. Early detection of sepsis-a role for network physiology? Crit Care Med 2016;44:e312-3.

31. Doyle SL, O'Neill LAJ. Toll-like receptors: from the discovery of NFkappaB to new insights into transcriptional regulations in innate immunity. Biochem Pharmacol 2006;72:1102-13.

32. Reys LG, Ortiz-Pomales YT, Lopez N, et al. Uncovering the neuroentericpulmonary axis: vagal nerve stimulation prevents acute lung injury following hemorrhagic shock. Life Sci 2013;92:783-92.

33. Balan KV, Kc P, Hoxha Z, Mayer CA, Wilson CG, Martin RJ. Vagal afferents modulate cytokine-mediated respiratory control at the neonatal medulla oblongata. Respir Physiol Neurobiol 2011;178:458-64.

34. Drexler SK, Foxwell BM. The role of toll-like receptors in chronic inflammation. Int J Biochem Cell Biol 2010;42:506-18.

35. Santello M, Bezzi P, Volterra A. TNF\$alpha\$ controls glutamatergic gliotransmission in the hippocampal dentate gyrus. Neuron 2011;69: 988-1001.

36. Kiss A, Tratsiakovich Y, Mahdi A, et al. Vagal nerve stimulation reduces infarct size via a mechanism involving the alpha-7 nicotinic acetylcholine receptor and downregulation of cardiac and vascular arginase. Acta Physiol 2017;221:174-81.

37. Andersson U, Antoine DJ, Tracey KJ. The functions of HMGB1 depend on molecular localization and post-translational modifications. J Intern Med 2014;276:420-4.

38. Tanner JA. Reversible blocking of nerve conduction by alternatingcurrent excitation. Nature 1962;195:712-3.

39. Kilgore KL, Bhadra N. Reversible nerve conduction block using kilohertz frequency alternating current. Neuromodulation J 2014;17: 242-54 discussion 254-255.

40. Bowman BR, McNeal DR. Response of single alpha motoneurons to highfrequency pulse trains. Firing behavior and conduction block phenomenon. Appl Neurophysiol 1986;49:121-38.

41. Klune JR, Dhupar R, Cardinal J, Billiar TR, Tsung A. HMGB1: endogenous danger signaling. Mol Med 2008;14:476-84. 